\title{
Retrospect and Inspiration from 5th International Symposium, Society for Research on the Cerebellum
}

\author{
Ying Shen
}

Published online: 7 April 2013

(C) Springer Science+Business Media New York 2013

\section{Introduction}

On September 23 and 24, 2012, 5th International Symposium of the Society for Research on the Cerebellum (SRC) was held at the Zhejiang University School of Medicine, Hangzhou, China. A proposal of having such symposium was submitted to SRC in 2011 and approved by the SRC council in early 2012. With the tremendous endeavor of organizing committee, all preparations were smoothly pushed forward for the success of the symposium. Here, we deeply appreciate the National Natural Science Foundation of China, Zhejiang University School of Medicine, Zeiss Microscope (Shanghai), Shanghai Zhengyu Equipment Co. Ltd, Thorlabs, Inc. (Shanghai), CIB (Shanghai), and Guangzhou Xuanming Bio-Tech Co. Ltd., for their financial supports that made the meeting feasible. We also give our sincere appreciation to all volunteers who greatly contributed to the symposium. They are Xin-Xin Wang, Liang Zhou, Chong-Yu Shao, Zhen Wang, Bo Chen, Ya-Jun Xie, and many more volunteers who cannot be listed due to limited space.

The organizing committee determined Jean Mariani is the chair; Ying Shen and Jian-Jun Wang are secretaries. A total of 70 researchers attended this symposium. Importantly, many Chinese scientists working in the cerebellar research field were attracted by the splendid conference speeches and registered in the meeting.

\footnotetext{
Y. Shen $(\bowtie)$

Department of Neurobiology, Key Laboratory of Medical Neurobiology of the Ministry of Health, Zhejiang Province Key Laboratory of Neurobiology, Zhejiang University School of Medicine, Hangzhou, People's Republic of China e-mail: yshen@zju.edu.cn
}

\section{Symposium Program}

Total 18 oral presentations were given in the symposium. Symposium 1: Cerebellar development and regeneration

1. Noriyuki Koibuchi. The role of thyroid hormone on functional organization in the cerebellum.

2. Izumi Sugihara. Peri- and postnatal development of cerebellar compartments.

3. Rachel M. Sherrard. How to build and rebuild a synapse in the cerebellum: formation and reformation of climbing fiber-Purkinje cell connectivity.

Symposium 2: Synaptic plasticity of cerebellum

1. Christian Hansel. Intrinsic plasticity in cerebellar Purkinje cells.

2. Sang Jeong Kim. TRPC channel underlies cerebellar long-term depression.

3. Wing Ho Yung. Effects of brain-derived neurotrophic factor (BDNF) on ionic plasticity of GABA transmission in the cerebellum: the role of KCC2.

4. Ying-Shing Chan. Neural plasticity during development of the vestibular system.

5. Ying Shen. $\mathrm{cPLA}_{2} \alpha /$ arachidonic acid is essential for parallel fiber-Purkinje cell synaptic LTP.

Symposium 3: Cerebellar functional analysis

1. Ho Yin Edwin Chan. Nucleolar stress in spinocerebellar ataxias.

2. Timothy Ebner. Purkinje cell simple spike discharge both predicts and responds to motor errors.

3. Donna Gruol. Neuroimmune regulation of neurophysiology in the cerebellum.

4. Jing-Ning Zhu. Hypothalamic modulation on cerebellar and vestibular motor control. 
5. Michael X. Zhu. Modulation of Purkinje cell firing in mouse cerebellum by p75-dependent Rac1 activation.

Symposium 4: Research on cerebellum diseases models

1. Mario Manto. The diversity of ideas on cerebellar involvement in movement.

2. Hidehiro Mizusama. Spinocerebellar ataxia type 31: a new RNA disease.

3. Yuchio Yanagawa. Transgenic approaches to the study of cerebellar GABAergic neurons.

4. Bei-Sha Tang. The role of transglutaminases in spinocerebellar ataxia: genetics and pathology.

5. Wen Wang. Memory traces for short- and long-term cerebellar motor learning.

Meanwhile, five posters were awarded "Masao Ito Prize".

1. De-Juan Wang, et al. Cytosolic phospholipase A2 alpha/ arachidonic acid signaling mediates depolarizationinduced suppression of excitation in the cerebellum.

2. Bo $\mathrm{Hu}$, et al. Prefrontal control of cerebellar motor learning.

3. Ming $\mathrm{Xu}$, et al. Abnormal cerebellar neurotrophin expression in autism patients and LPS-treated rat model.

4. Lei Yu, et al. Orexin selectively excites cerebellar nuclear projection neurons and promotes motor behaviors.

5. Xiao-Yang Zhang, et al. Orexin regulates excitability and sensitivity of the medial vestibular nucleus neurons via nonselective cation channels and $\mathrm{Na}^{+} / \mathrm{Ca}^{2+}$ exchangers.

\section{Summary of Symposium}

In this symposium, lectures from three speakers covered the field of the cerebellar development and regeneration. Noriyuki Koibuchi from Gunma University summarized novel roles of thyroid hormone in the morphogenesis and functional organization of cerebellum as well as motor behaviors. The work from Sugihar Izumi (Tokyo Medical and Dental University) combined immunohistochemistry and $3 \mathrm{D}$ reconstruction techniques and described compartmentalization of Purkinje cells during the cerebellar development. Particularly, they used multiple molecular markers to label subsets of Purkinje cells at peri- and postnatal stages and mark these cells in a much greater detail. Rachel $\mathrm{M}$. Sherrard (University Pierre et Marie Curie) opened a new sight by showing that the maturation of Purkinje cells and climbing fiber influences the capacity of synaptic formation and re-innervation at climbing fiber-Purkinje cell synapses. They showed that BDNF/TrkB, PSA-NCAM, and ROR are the underlying mechanisms.

As always, plasticity is one interesting topic in the cerebellum, which is the central process unit in many motor behaviors and conditioning reflexes. In this symposium, several speakers presented their recent findings in cerebellar plasticity. Sang Jeong Kim (Seoul National University) pushed forward the mechanism of well-studied parallel fiber-Purkinje cell long-term depression (LTD) by showing that blocking TRPC3 channels in Purkinje cell abolish LTD induction. Oppositely, Ying Shen (Zhejiang University) presented functions of $\mathrm{cPLA}_{2} \alpha /$ arachidonic acid/2-AG signaling in parallel fiber-Purkinje cell long-term potentiation. Wing Ho Yung (Chinese University of Hong Kong) displayed the modulation of BDNF signaling on GABAergic synaptic transmission in cerebellar Purkinje neurons, which includes KCC2. Christian Hansel (Chicago University) summarized their several contributions in the intrinsic plasticity in Purkinje cells.

It is encouraged that more efforts are currently devoted to the functional analysis of cerebellar behavior, which were nicely shown in this symposium. Ying-Shing Chan (University of Hong Kong) presented a nice study on the topographic map of inferior olive neurons during horizontal head movements, providing another important piece of information to understand the fine regulation of sensorimotor coordination. Ho Yin Edwin Chan (Chinese University of Hong Kong) found that CAG expansion induces nucleolar stress in spinocerebellar ataxia and is involved in Huntington disease. Timothy Ebner (University of Minnesota) reported that simple spikes of Purkinje cells predict and respond to motor errors. Their data show that error parameters are represented in the simple spike activities, besides complex spike that has been accepted for a long period. Donna Gruol (Scripps Institute) showed an unexpected function of IL-6 on the expression of neuronal proteins and mGluR1-activated $\mathrm{Ca}^{2+}$ signaling in Purkinje cells. Their results indicate that the disorder of cerebellar functions is likely to be associated with an elevated IL-6 in the cerebellum. Jing-Ning Zhu (Nanjing University) reported the somatic-nonsomatic integration between the hypothalamus and cerebellum and vestibular nucleus through histaminergic and orexinergic systems.

Several speakers presented recent research progresses in cerebellum-related diseases. Mario Manto (Université Libre de Bruxelles) attempted to obtain a "consensus" on diverse theories in clinical cerebellar pathogenesis that regards the nature of basic operations of motor control. SCA31 is a neurodegenerative disease characterized by pure cerebellar ataxia with degenerating Purkinje cells. Hidehiro Mizusawa (Tokyo Medical and Dental University) reported that RNA foci formation containing (UGGAA) ${ }_{n}$ may be a hallmark of SCA31 neurodegeneration. Similar observations were also presented by Bei-Sha Tang (Xiangya Hospital) who presented a talk entitled "The role of transglutaminases in spinocerebellar ataxia: genetics and pathology."

Oral and poster reports presented in this symposium were excellent. The scientific communications were very active. 
In this special issue, we have chosen 14 lectures to share with the Cerebellum readers. The videos of all lectures will be online in future.

\section{Future Perspectives}

This symposium is second SRC annual meeting held in Asia. We are pleased to notice that more Asian scientists, who came from Japan, Korea, China Hong Kong, and China mainland, contributed greatly to this meeting, which will promote the development of SRC in Asia. Pleasantly, more than 40 Chinese mainland scientists attended this meeting. China's economy has been booming since twenty-first century, accompanying by outstanding achievements in sports and arts. Now, Chinese people realize that research in science and technology is one of the most important foundations for a nation. As the result, national and local grants for basic and clinical scientific researches have dramatically increased year by year. Meanwhile, research papers published by Chinese scientists have also incredibly increased. We are very optimistic that the cerebellar research will also grow up rapidly in the near future.

There are still many issues regarding SRC symposium and cerebellar research. One of them is how to appeal for more researchers to attend the meeting. It may be a good idea to have the symposium with a longer period or being held as a satellite meeting in conjunction with the Federation of European Neuroscience Symposium or the Annual Meeting of Society for Neuroscience. The nature is that we should call on more re- searchers to actively join the cerebellar research. To achieve this goal, researchers should expand novel and more comprehensive studies to explore the functions of the cerebellum in more disease models, but not only ataxia. It is exciting that some lectures in this symposium have begun to explore this direction.

As the ending, I am happy to share one photo with all readers, in which some attendees are present.

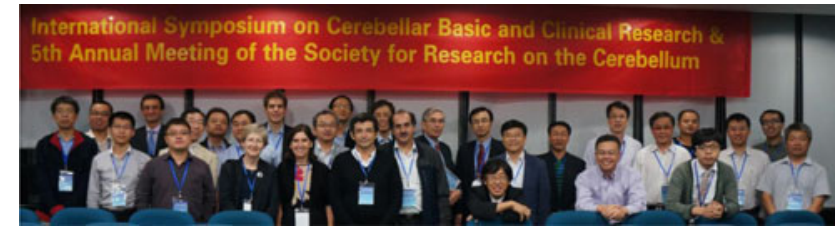

From left to right: Yuqing Li, Ming Xu, Hao Chen, Mario Manto, Ying Shen, Izumi Sugihara, Michael X. Zhu, Delai Qiu, Rachel M. Sherrard, Christian Hansel, Donna Gruol, Yuchio Yanagawa, Sang Jeong Kim, Jean Mariani, Ying-Shing Chan, Hassan Marzban, Timothy Ebner, Hidehiro Mizusawa, Noriyuki Koibuchi, Joo Rhyu Im, Jie Na, Jian-Hong Luo, Wing Ho Yung, Jian-Jun Wang, Wen Wang, Hyun-wook Kim, Jin-Ning Zhu, Xiao-Yang Zhang, and Bing Xu.

Acknowledgments SRC committee and local organizing committee of 5th SRC international symposium thank all researchers who attended the symposium. We are grateful for all companies and organizations that supported the symposium. We would like thank all volunteers for their wonderful contributions. This symposium is supported by NSFC (No. 31210303038 to Y.S.).

Conflict of interest There are no conflicts of interest in this submission. 